\title{
SPARTA: Stable and Efficient Spectrum Access in Next Generation Dynamic Spectrum Networks
}

\author{
Lili Cao and Haitao Zheng \\ Department of Computer Science \\ University of California, Santa Barbara, CA 93106 U.S.A
}

\begin{abstract}
Future wireless infrastructure networks will dynamically access spectrum for maximum utilization. However, the fundamental challenge is how to provide stable spectrum access required for most applications. Using dynamic spectrum access, each node's spectrum usage is inherently unpredictable and unstable. We propose to address this challenge by integrating interference-aware statistical admission control with stabilitydriven spectrum allocation. Specifically, we propose to proactively regulate nodes' spectrum demand to allow efficient statistical multiplexing while minimizing outages. Admitted nodes coordinate to adapt instantaneous spectrum allocation to match timevarying demand. While the optimization problem is NP-hard, we develop computational-efficient algorithms with strong analytical guarantees. Experimental results show that the proposed approach can provide stable spectrum usage while improving its utilization by 80-100\% compared to conventional solutions.
\end{abstract}

\section{INTRODUCTION}

Continuous wireless innovation and development require easy and reliable access to radio spectrum. However, existing regulations allocate spectrum to each technology/network statically in peak-demand based long-term leases. Overtime, most of spectrum has been assigned but their utilization is as low as $10-15 \%$. The ideal solution is dynamic spectrum access (DSA). Under this new model, wireless nodes request spectrum dynamically, adapting to time-varying demand. By multiplexing spectrum usage across time and space, DSA can significantly improve its utilization.

While providing efficient spectrum utilization, DSA, however, faces a critical problem of unstable spectrum usage. In DSA networks, wireless nodes coexist and must share spectrum to avoid interference [4]. Because of network and traffic dynamics [14], their achieved spectrum usages fluctuate and are inherently unpredictable and unstable. Frequent, unpredictable fluctuations in spectrum usage will not only severely disrupt communication links, but also lead to frequent service interruptions. To meet critical service agreements, service providers are forced to significantly over-provision the network, leading to unnecessary financial penalties and underutilization of spectrum.

In this paper, we seek to achieve efficient and stable spectrum access, targeting commercial wireless infrastructure networks such as WiMAX. We consider a large deployment of wireless access points (APs) in a city or large campus. Each AP has time-varying spectrum demand, and must obtain desired spectrum consistently and continuously to meet critical service agreements. The spectrum allocation is interferencedriven. APs in close proximity interfere with each other and cannot use the same spectrum concurrently, while wellseparated APs can reuse the same spectrum. The problem is particularly challenging because of the combinatorial interference constraints.

We propose SPARTA, a new DSA architecture to provide efficient and stable spectrum usage by integrating proactive planning with reactive adaptation. First, SPARTA introduces a novel statistical admission control algorithm to proactively prevent congestion of spectrum demand while addressing interference constraints. Specifically, SPARTA determines the volume of supported demand at each AP based on its distribution statistics and local interference condition. As a result, SPARTA can exploit both time and spatial multiplexing to maximize spectrum utilization while providing a probabilistic guarantee of usage stability. Next, SPARTA introduces an efficient allocation algorithm to quickly adapt admitted APs' spectrum usage to match their time-varying demands. Together, these two components achieve stable spectrum usage while achieving efficient spectrum utilization.

SPARTA's proactive planning and reactive adaptation components can be placed at different network entities to enhance scalability. A central server can perform proactive planning periodically to regulate APs' long-term spectrum demand, while admitted APs coordinate among themselves in a distributed manner to adapt instantaneous spectrum allocation.

Our work makes three major contributions:

- Interference-aware statistical admission control - We develop an effective-rate based demand shaping algorithm to determine which APs to admit and the volume of their spectrum demands under a given outage rate. We build analytical bounds on the algorithm efficiency in terms of its distance to the optimal solution.

- Stability-driven distributed spectrum allocation - We propose a computational-efficient algorithm to adapt instantaneous spectrum allocation to time-varying demand. APs coordinate and perform local actions to optimize the global spectrum allocation. While the optimization problem is NP-hard, the proposed algorithm has polynomial complexity and can achieve the level of stability promised by the admission control component.

- Integrated evaluation - We examine SPARTA using both network simulations and analytical evaluations. We also explore the impact of traffic statistics, network topology and admission granularity. Both experimental and ana- 

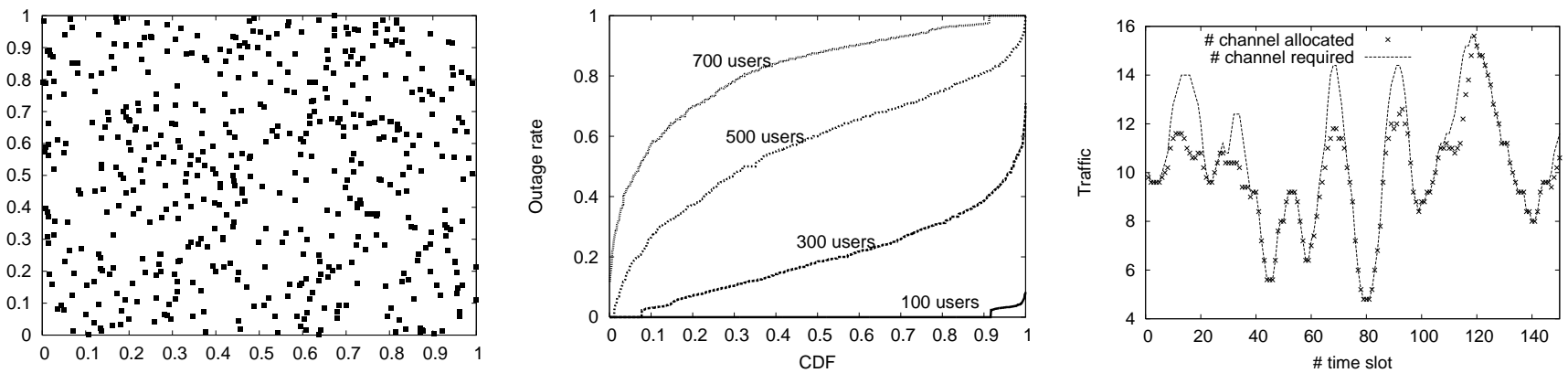

Fig. 1. A dynamic spectrum access system: (left) Network topology: APs are randomly located in a wide area, with uniform traffic statistics. (center) The CDF of node spectrum outage rate at different network size. (right) A sample trace of spectrum demand and assignment at a node over time.

lytical results verify that SPARTA achieves efficient and stable spectrum access, and significantly outperforms the conventional peak-rate based mechanism.

\section{BACKGROUND AND RELATED WORK}

In this section, we briefly present the features of dynamic spectrum access, the problem of unstable spectrum access and related work.

\section{A. Dynamic Spectrum Access}

In DSA systems, nodes do not have statically assigned spectrum, but dynamically compete for spectrum based on current demand. There are multiple complementary ways to implement DSA systems, applicable to different scenarios. Nodes can use a single spectrum band but adjust transmit power to minimize interference [7], [11]. Alternatively, conflicting nodes can use different orthogonal channels to avoid interference, and optimize channel allocations to maximize a predefined system utility [3], [4], [19], [20], [21], [22]. A recent survey [2] provides an overview of existing solutions.

The Problem of Unstable Spectrum Access. Existing DSA proposals assume static network topology and spectrum demand, optimizing instantaneous system utility such as throughput and fairness. Across time, nodes access spectrum opportunistically, making it difficult to obtain desired spectrum consistently. To further explore this problem, we examine individual node's spectrum usage across time by deploying a large set of nodes (APs) randomly in a wide-area ( Figure 1(left)), each with time-varying spectrum demand. We allocate spectrum channels to maximize the networkwide proportional fairness [4]. Figure 1(center) examines the cumulative distributed function (CDF) of the spectrum outage where a node experiences an outage if its assigned spectrum is less than its demand. Not surprisingly, individual outage rate increases significantly with the network size. Figure 1(right) plots a sample trace of the spectrum allocation and demand at a particular node over time. Clearly, spectrum outage becomes a norm rather than an exception.

\section{B. Related Work}

The problem of spectrum allocation is often modeled as resource scheduling problems [12]. The system must allocate spectrum channels to active nodes/links to avoid interference. However, existing proposals on spectrum/channel allocation [3], [4], [12], [19], [20], [21], [22] assume static spectrum demand and do not address stability. Recent work [23], [24] proposes algorithms to dynamically allocate spectrum to match demands. However, it is best-effort and does not provide any stability guarantee. Our work differs from these existing works by integrating admission control with dynamic spectrum allocation to achieve stable and efficient spectrum usage.

Our proposed approach differs from conventional statistical admission control proposals [17] by being interference-aware. Most proposals are designed for routers or cellular networks where traffic multiplexing happens at a single point (routers or base stations). In this case, the optimization problem is subject to a simple linear constraint, where the total demand does not exceed the route/base station capacity [15], [16]. Motivated by these results, our proposal focuses on the spectrum allocation problem with a set of combinatorial interference constraints. Our unique contribution is to find efficient solutions to the corresponding NP-hard problem. To our best knowledge, we are the first to address the stability problem in DSA systems.

\section{Achieving Stable Spectrum Access}

In this section, we first define the problem of providing stable dynamic spectrum access, and then present the highlevel concept of SPARTA.

\section{A. Problem Definition}

We consider a large infrastructure network with $N$ APs and $M$ channels in a time horizon of $[0, T]$. In the following, the notions of APs and nodes are interchangeable. To make the problem tractable, we make several assumptions. First, using OFDM, the spectrum is divided into a set of orthogonal channels. Second, nodes can utilize multiple non-continuous channels concurrently. Third, we focus on channel assignment and assume fixed transmit power. The interference condition is modeled by a set of combinatorial constraints [3], [13], [21] among nodes. Finally, although nodes can modify their spectrum usage at any time, we assume the time is divided into small slots and nodes can only modify spectrum usage at the beginning of each slot.

We introduce the following notations: 


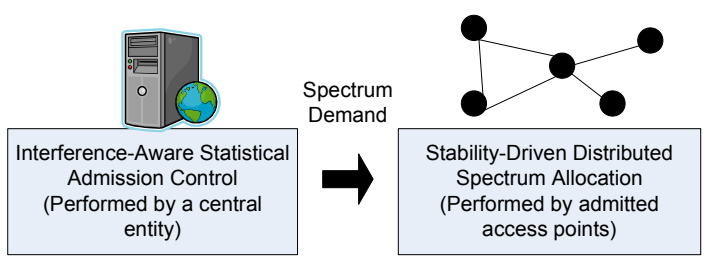

Fig. 2. High-level structure of the proposed approach. A spectrum server admits nodes and determines their spectrum demand. Admitted nodes coordinate to determine spectrum allocation in each time slot matching their time-varying demand.

- Spectrum demand $D_{n}(t)$ : the amount of spectrum node $n$ requests at time $t$

- Demand shaping $\Re_{n}($.$) : the shaping determined at the$ time of admission. The admitted demand at time $t$ is $\Re_{n}\left(D_{n}(t)\right)$, and $0 \leq \Re_{n}\left(D_{n}(t)\right) \leq D_{n}(t)$.

- Spectrum allocation $A_{n}(t)=\sum_{m=1}^{M} a_{n, m}(t)$ : The amount of spectrum $n$ gets at time $t$, where $a_{n, m}(t) \in\{0$, $1\}$ and $a_{n, m}(t)=1$ means channel $m$ is allocated to $n$.

- Interference constraints $c_{n, k, m}$ : the conflict constraint between node $n$ and $k$ on channel $m . c_{n, k, m}=1$ means node $n$ and $k$ should not use channel $m$ at the same time. That is, $a_{n, m}(t) \cdot a_{k, m}(t)=0$, if $c_{n, k}=1$. For simplicity, we assume that the channels have identical interference characteristics, and hence $c_{n, k, m}=c_{n, k}$.

The optimization problem can be defined as: given $\left\{c_{n, k}\right\}_{N \times N}$ and the statistical distribution of $D_{n}(t)$, find $\left\{\Re_{n}(.)\right\}_{N}$ and $\left\{a_{n, m}(t)\right\}_{N \times M}$, to

$$
\begin{aligned}
& \max \sum_{n=1}^{N} \mathbb{U}\left(\frac{1}{T} \sum_{t=0}^{T} \Re_{n}\left(D_{n}(t)\right)\right) \quad \text { subject to } \\
& \operatorname{Prob}\left\{A_{n}(t)<\Re_{n}\left(D_{n}(t)\right\} \leq \theta_{n},\right. \\
& a_{n, m}(t) \cdot a_{k, m}(t)=0, \text { if } c_{n, k}=1, \\
& \forall n, k \in[1, N], m \in[1, M]
\end{aligned}
$$

where $\theta_{n}$ represents the maximum outage rate at node $n$, $\mathbb{U}($.$) represents the utility function used by the admission$ control module. In this paper, our goal is to maximize the total admitted demand, where $\mathbb{U}(x)=x$. Our results can be easily extended to other utility functions.

The above problem can be divided into two sub-problems: 1) at time 0 , defining spectrum shaping $\left\{\Re_{n}(.)\right\}_{N}$, and 2) from time 0 to $T$, performing instantaneous spectrum allocation in each time slot to find $\left\{a_{n, m}(t)\right\}_{N \times M}$. Because of the combinatorial interference constraints, both sub-problems are NP-hard.

\section{B. A Simple Solution: Peak Demand based Static Access}

We can simplify the above problem by using the peak demand based admission. Let $D_{n}^{\max }$ denote a node $n$ 's peak demand over time, $D_{n}^{\max }=\max _{t \in[0, T]} D_{n}(t)$. The system treats each (bursty) demand as if it is a constant traffic at a rate of $D_{n}^{\max }$ and assigns spectrum to match its peak demand. The system can also shape the peak demand of $n$ by a factor of $r_{n}$. Therefore, the problem reduces to

$$
\begin{aligned}
& \max \sum_{n=1}^{N} \mathbb{U}\left(r_{n} D_{n}^{\max }\right)=\sum_{n=1}^{N} \mathbb{U}\left(\sum_{m=1}^{M} a_{n, m}\right) \quad \text { subject to } \\
& a_{n, m} \cdot a_{k, m}=0, \text { if } c_{n, k}=1, \forall n, k \in[1, N], m \in[1, M] .
\end{aligned}
$$

This problem becomes a throughput maximization problem, which can be solved by using efficient heuristics [21]. Despite of the algorithm simplicity, most assigned spectrum sits unused overtime, leading to severe under-utilization of spectrum. This observation motivates us to search for a new solution to cope with the time-varying demand.

\section{The SPARTA Architecture}

As shown in Figure 2, SPARTA consists of two components, addressing each of the two sub-problems.

- A central entity performs interference-aware statistical admission control to regulate spectrum demand and prevent outages. Instead of using the peak demand, this components operates on the statistical distribution of the spectrum demand at each AP, and exploiting time and spatial multiplexing to admit more APs into a system than is possible when assuming that each admitted AP is fully loaded with its peak demand.

- Admitted APs perform stability-driven distributed spectrum allocation to determine instantaneous spectrum usage to match their time-varying demands. This component applies a distributed, low-complexity algorithm for quick adaptation. With sufficient backbone bandwidth, this algorithm can also run at a central entity who collects demands in each time slot and allocates spectrum accordingly.

Overall, the unique contributions of SPARTA are the development of computational-efficient algorithms for both admission control and spectrum allocation, and the integration of both components to maximize stability and utilization in spectrum usage. In the following two sections, we will describe these two components in details.

\section{INTERFERENCE-AWARE STATISTICAL ADMISSION CONTROL}

Our design is motivated by prior work on statistical admission control using effective rate [15], [16]. However, because of the combinatorial interference constraints, the original solution cannot be directly applied to DSA networks. In this section, we propose an interference-aware statistical admission control that exploits both the time and spatial multiplexing nature of input spectrum demand. Our work assumes perfect knowledge of the statistical model of each AP's spectrum demand and the global interference constraints. We will discuss the practical implication in Section VII.

\section{A. Background on Effective Rate}

In wired networks, the problem of admitting bursty flows has been addressed by a concept known as the effective rate [1], [6], [15], [16]. By computing an appropriate effective 
rate of a flow, routers can treat the flow (with bursty traffic) as if it is a constant traffic at this effective rate during the active period of the flow. Routers then determine the feasibility of admitting a set of flows by ensuring the sum of their effective rate is no more than the total available bandwidth subtracting a factor. Similar concept has been applied to wireless networks where a base station determines whether to admit a user.

Let $X_{i}(1 \leq i \leq N)$ represent a random variable, whose value is the amount of bandwidth requested by node $i$ in a time slot. The router admits all $N$ nodes if

$$
\operatorname{Prob}\left\{\sum_{i=1}^{N} X_{i}>C\right\} \leq e^{-\gamma},
$$

where $e^{-\gamma}$ represents the outage rate, a system parameter, and $C$ represents the total amount of bandwidth. Define the effective rate [15] of node $X_{i}$ as:

$$
\alpha_{i}(s)=\frac{1}{s} \log E\left[e^{s X_{i}}\right], \quad 0<s<+\infty .
$$

Since $\forall s, \operatorname{Prob}\left\{\sum_{i=1}^{N} X_{i}>C\right\} \leq e^{s\left(\sum_{i} \alpha(s)-C\right)}$, it is easy to show that (3) reduces to a deterministic measure

$$
\text { if } \exists s, \sum_{i=1}^{N} \alpha_{i}(s) \leq C-\frac{\gamma}{s} .
$$

While the original design of effective rate assumes independent traffic, many have applied and validated the concept using real network traffic traces [9], [10].

\section{B. Effective Rate based Spectrum Demand Shaping}

The original effective-rate design relies on the linear constraints of (3). However, in DSA systems, the interference constraints (described in (2)) are combinatorial and non-linear. To address this challenge, we propose to use linear constraints to approximate the original non-linear interference constraints, and perform demand shaping on each constraint (shown in Table I). We must design such approximation judiciously to avoid producing weaker constraints that lead to interference or overly restrictive constraints that degrade spectrum utilization.

We propose a linear approximation using the geometric order. We introduce the notion of left of. Let two nodes $i$ and $j$ locate at coordinates $\left(x_{i}, y_{i}\right)$ and $\left(x_{j}, y_{j}\right)$. Node $i$ is to the left of node $j$ if $x_{i}<x_{j}$. If $x_{i}=x_{j}$, then the node with the smaller index is considered to be to the node to the left. Using this geometric order, we propose Node-L Constraints. Let $\hat{A}_{n}(t)$ be the total amount of spectrum allocated to node $n$ under this new constraint. The new constraint is

$$
\hat{A}_{n}(t)+\sum_{k \in I_{n}} \hat{A}_{k}(t) \leq 1, \forall n .
$$

where $I_{n}$ is the set of conflict peers of $n$ in the original constraint who are left of $n$. This approximation reduces the original combinatorial constraints into $N$ linear constraints.

Our prior work [8] has shown that the Node-L constraints are stricter than the original combinatorial constraints and hence lead to a conflict-free but sub-optimal spectrum allocation. Further, analytically this sub-optimal solution is at most a distance of 3 from the optimal solution.
TABLE I

DERIVING EFFECTIVE-RATE BASED ADMISSION CRITERIA VIA LINEAR CONSTRAINTS APPROXIMATION.

\begin{tabular}{ccc} 
& Linear & Effective Rate-based \\
Original & Interference Constraints & Admission Criteria \\
Interference & $\sum_{i \in I_{1}} X_{i} \leq C$ & $\exists s_{1}, \sum_{i \in I_{1}} \alpha_{i}\left(s_{1}\right) \leq C-\frac{\gamma}{s_{1}}$ \\
Constraints & $\sum_{i \in I_{2}} X_{i} \leq C$ & $\exists s_{2}, \sum_{i \in I_{2}} \alpha_{i}\left(s_{2}\right) \leq C-\frac{\gamma}{s_{2}}$ \\
& $\sum_{i \in I_{K}} X_{i} \leq C$ & $\exists s_{K}, \sum_{i \in I_{K}} \alpha_{i}\left(s_{K}\right) \leq C-\frac{\gamma}{s_{K}}$ \\
\hline
\end{tabular}

Under the Node-L constraints, the spectrum shaping problem can be defined as

$$
\begin{array}{ll}
\max _{\left\{\Re_{n}\right\}} \sum_{n=1}^{N} \mathbb{U}\left(\frac{1}{T} \sum_{t=0}^{T} \Re_{n}\left(D_{n}(t)\right)\right) & \text { subject to } \\
\exists s_{n}, \quad \sum_{i \in I_{n}} \alpha_{i}^{\Re_{i}}\left(s_{n}\right) \leq C-\frac{\gamma}{s_{n}}, \quad \forall 1 \leq n \leq N, &
\end{array}
$$

where $C$ is the total volume of spectrum, and $\alpha_{i}^{\Re_{i}}($.$) is the$ effective rate of the demand with shaping $\Re_{i}$.

We note that using Node-L constraints, each individual node's outage rate is bounded by the outage rate of the constraint. And we can directly define $\gamma=-\ln \left(\min _{n} \theta_{n}\right)$. This is because each node $n$ maps to a constraint in the above problem (7), which maps to a constraint in (6). By properly allocating channels (as described in Section V), a node only experiences an outage if the corresponding constraint is violated. This means a node's individual outage rate is bounded by $e^{-\gamma}$, the probability of any constraint being violated.

For simplicity we consider two admission policies:

- Binary shaping where $\Re_{n}(x)=r_{n} x$ and $r_{n} \in\{0,1\}$, i.e., a node is either fully admitted or declined.

- Continuous shaping where $\Re_{n}(x)=r_{n} x$ and $r_{n} \in[0,1]$, e.g., a node can be partially admitted.

For both policies, solving (7) is still challenging as it requires complex non-linear optimization. Under arbitrary demand statistics, there is generally no closed form for $\alpha(s)$. Further, the fundamental challenge is how to determine $r_{n}$ and $s_{n}$. In particular, given $\alpha($.$) and r_{n}$, computing $s_{n}$ in each constraint is already computationally expensive. Yet for dynamic spectrum access, long delay in admission can severely degrade the system performance. The interdependency between $r_{n}$ and $s_{n}$ makes the whole optimization even more challenging.

To make (7) tractable, we propose to choose $s_{n}$ based on APs' demand statistics. In the following, we investigate the impact of parameter $s_{n}$ and then propose analytical results on deriving sub-optimal $s_{n}$ values under different demand statistics. After choosing $s_{n}$, we develop efficient solutions to find sub-optimal $r_{n}$ values.

\section{Searching for the Optimal $s$}

We simplify the problem by using a uniform $s$, i.e. $s_{n}=s$, $\forall n$. We show that the use of uniform $s$ is optimal under uniform node traffics for binary admission. Next, when APs have non-uniform demand statistics, we present several heuristics to choose the uniform $s$. Finally, we analyze the impact of using a uniform $s$. 
The following three theorems assume binary demand shaping.

\section{1) Uniform Traffic Statistics}

Theorem 1 If nodes have uniform demand patterns, then there exists a $0<s<+\infty$ such that the system utility can be maximized by choosing $s_{n}=\dot{s}, \forall n$.

The proof of Theorem 1 is in the Appendix. From the proof we can see that the optimal value of $s$ does not depend on the topology of the network.

2) Non-Uniform Traffic Statistics

Theorem 2 Let $t_{1}, t_{2}, \ldots, t_{J}$ represent the set of demand statistics among APs in the network. Let $\dot{s}_{j}$ represent the optimal $s$ if every node has demand statistics $t_{j}$. If every constraint must use the same $s$, then the optimal uniform $s$ is bounded:

$$
\min \left\{s_{1}, s_{2}, \cdots s_{J}\right\} \leq s_{\text {opt }} \leq \max \left\{s_{1}, s_{2}, \cdots s_{J}\right\} .
$$

The proof of Theorem 2 is in the Appendix.

Using Theorem 2, we have two heuristics to choose a uniform $s$. We can always use numerical search in the above range. Or, we can choose $s$ based on the average demand statistics. In particular, when the demand follows the On/Off traffic model, we choose $s$ as if traffic is uniform with the average peak rate $h$ and average mean rate $m$. We will compare these heuristics in Section VI.

The following theorem shows that the performance degradation for choosing a uniform $s$ under non-uniform spectrum demands is bounded.

Theorem 3 Let $t_{j}$ and $s_{j}$ as defined in Theorem 2. Define the performance degradation function

$$
\begin{aligned}
g_{j}(s) & =\frac{C-\gamma / s}{\alpha_{j}(s)} / \frac{C-\gamma / \dot{s}_{j}}{\alpha_{j}\left(\dot{s}_{j}\right)} \\
g(s) & =\min _{j} g_{j}(s), \quad g_{j}(s) \leq 1 .
\end{aligned}
$$

If a utility $U$ can be achieved by choosing possibly nonuniform $s_{j}$, then $U$ can also be achieved by choosing a uniform $s_{j}=s$ given a total spectrum capacity of $C / g(s)$.

Theorem 3 means we can bound the degradation of using uniform $s$ under non-uniform traffic by examining the impact of $s$ under uniform traffic. The proof can be found in [5].

\section{Fast Algorithm for Continuous Demand Shaping}

After determining a uniform ś, we develop an efficient optimization mechanism to determine $\Re_{n}$. Using the On/Off traffic model as an illustration, we describe the algorithm for continuous shaping in the following, and the algorithm for binary shaping in Section IV-E.

Assuming the spectrum demand follows the On/Off model, we can compute the effective rate of AP $i$ as:

$$
\alpha_{i}^{r_{i}}(s)=\frac{1}{s} \log \left[1+\frac{m_{i}}{h_{i}}\left(e^{s \cdot h_{i} \cdot r_{i}}-1\right)\right],
$$

where $h_{i}$ is the peak rate, $m_{i}$ is the mean rate, and $r_{i}$ is the demand shaping factor. The optimization problem reduces to

$$
\begin{aligned}
& \max _{\left\{r_{n}\right\}} \sum_{n=1}^{N} \mathbb{U}\left(r_{n} m_{n}\right) \quad \text { subject to } \\
& \exists s_{n}, \quad \sum_{i \in I_{n}} \alpha_{i}^{r_{i}}\left(s_{n}\right) \leq C-\frac{\gamma}{s_{n}}, \quad \forall 1 \leq n \leq N,
\end{aligned}
$$

Let $x_{i}=\alpha_{i}^{r_{i}}(s)$. We can calculate $r_{i}$ from $x_{i}, r_{i}=\mathbb{F}^{i}\left(x_{i}\right)$, and reduce (10) to

$$
\begin{aligned}
& \max _{\left\{x_{n}\right\}} \sum_{n=1}^{N} \mathbb{U}\left(\mathbb{F}^{n}\left(x_{n}\right) m_{n}\right) \quad \text { subject to } \\
& \sum_{i \in I_{n}} x_{i} \leq C-\frac{\gamma}{s}, \quad \forall 1 \leq n \leq N,
\end{aligned}
$$

Because $\mathbb{F}^{n}($.$) is non-linear, solving (11) can be com-$ putationally expensive. We propose a low-complexity local search algorithm using the concept of "Feed Poverty" [4]. The algorithm runs iteratively. In iteration $k$, the algorithm will

1) Choose node $i$, increase its effective rate: $x_{i}^{k}=x_{i}^{k-1}+$ $\Delta x$.

2) Adjust the effective rate of other nodes who share the same constraints with $i$ until all the constraints are satisfied.

3) If the total system utility increases, apply this improvement, go to iteration $k+1$. Otherwise, discard the improvement, go to iteration $k+1$.

The algorithm terminates if no more local modifications can improve the system utility. Because the system utility is finite and each improvement increases the utility, the algorithm is guaranteed to terminate. Clearly, the algorithm performance depends heavily on the choice of node $i$ in step 1. One must carefully pick $i$ based on the utility function $\mathbb{U}($.$) . For example,$ if the goal is to maximize proportional fairness or max-min, the system should choose the node with the worst $r_{n} m_{n}$ [4].

In addition, the algorithm performance also depends on how to adjust the effective rate of other nodes to satisfy all the constraints. We propose to use the following two policies:

(1) Proportionally amortized decrease - For each constraint $I$ which contains $x_{i}$, if increasing $x_{i}$ violates $I$, the algorithm must decrease the effective rate of other nodes in $I$. A simple way is to decrease every other node by $\frac{\Delta x}{n-1}$, where $n$ is the number of nodes in the constraint. However, this mechanism requires every node's effective rate to be at least $\frac{\Delta x}{n-1}$. Instead, we propose to use proportionally amortized decrease which amortizes the total amount of the decrease proportionally among nodes in the same constraint. Assuming the violated constraint is $\sum_{n \in I} x_{n} \leq C^{\prime}$, and $i^{*} \in I$ is the node to be improved at stage $k$, our adjustment is defined by

$$
x_{n}^{k}=x_{n}^{k-1}\left(1-\frac{\sum_{n \in I} x_{n}^{k-1}-C^{\prime}+\Delta x}{\sum_{n \in I \backslash\left\{i^{*}\right\}} x_{n}^{k-1}}\right), \forall n \in I \backslash\left\{i^{*}\right\}
$$

(2) Tightest constraint first - Because each node $i$ participates in multiple constraints, increasing $x_{i}$ can violate multiple 
constraints. We need to optimize the ordering of constraint adjustments to avoid unnecessary ping-pong effect. We propose to adjust constraints in decreasing order of their tightness. We define the tightness of a violated constraint $\sum_{i \in I} x_{i} \leq C$ as

$$
\operatorname{Tightness}(I)=\frac{\sum_{i \in I} x_{i}-C}{\sum_{i \in I \backslash\left\{i^{*}\right\}} x_{i}} .
$$

\section{E. Greedy Algorithm for Binary Demand Shaping}

Using binary shaping, the value of $r_{n}$ is either 0 or 1 . We propose a simple sequential strategy: starting from an empty admission, iteratively admit nodes one by one. At each iteration, randomly choose a node and check whether admitting it will violate the constraints. If not, admit it, otherwise, select another node. The process repeats until no nodes can be admitted.

\section{Stability-Driven Distributed CoOrdination}

After determining $\Re_{n}($.$) , the optimization problem re-$ duces to determining instantaneous spectrum allocation in each time slot $t,\left\{a_{n, m}(t)\right\}_{N \times M}$, to match spectrum usage to time-varying demand. Specifically, given $\left\{c_{n, k}\right\}_{N \times N}$, $\left\{\Re_{n}\left(D_{n}(t)\right)\right\}_{N}$, find $\left\{a_{n, m}(t)\right\}_{N \times M}$, such that

$$
\operatorname{Prob}\left\{A_{n}(t)<\Re_{n}\left(D_{n}(t)\right)\right\} \leq \theta_{n},
$$

subject to $a_{n, m}(t) \cdot a_{k, m}(t)=0$, if $c_{n, k}=1, \forall m$.

Similarly, because of the combinatorial interference constraints, the problem is NP-hard. More importantly, the solution must be computational-efficient to provide quick adaptation to varying demands. We propose a low-complexity distributed algorithm where admitted APs determine spectrum allocation using local coordination [25]. Using the same geometric ordering in the admission control phase, each AP $n$ applies the following procedure to choose spectrum channels.

1) Mark all the channels that are used by its neighbors who are in front of $n$ in the ordering as "unavailable"; mark all the channels that are used by its neighbors who are behind $n$ in the ordering as "busy"; mark the rest as "idle";

2) Select channels from the set of "idle" channels. If the set is not sufficient to meet $n$ 's demand, take channels from the set of "busy" channels. Neighboring nodes who are using these "busy" channels will exit from these channels and mark them as "unavailable".

3) Stop if $n$ 's demand is satisfied or if the "idle" and "busy" sets become empty.

The following properties show that the proposed algorithm can guarantee stability as promised by the admission control component, and that the algorithm complexity is bounded.

Property 1 The above algorithm guarantees that each node's individual outage rate is lower than the theoretical bound $\theta_{n}$.

This is because an AP $n$ experiences an outage only if the constraint $I_{n}$ (cf. (6)) is violated. Because the probability of violation is bounded by $\theta_{n}$, so is the outage rate of $n$.

Property 2 The expected total number of spectrum adaptation is upper-bounded by $O\left(N^{2}\right)$ if the ordering of adaptation is random, and upper-bounded by $N$ if the ordering is optimized. (The proof follows a similar concept of those in [25] and hence omitted due to space limit.)

\section{EXPERIMENTAL RESULTS AND DISCUSSIONS}

We now evaluate SPARTA using network simulations. We randomly deploy APs in an $1 \times 1$ area. We use a simple distance based measure to determine interference constraints, i.e. two nodes conflict if their distance is within $d=0.2$. While this is a simple approach to produce the conflict condition $a_{n, k}$, it will not limit the scope of our proposed approach. By default, we assume that nodes' demand follows the On/Off model with mean $m$ and peak rate $h$. We also examine the impact of having non-On/Off models. We use the utilization-based system utility function, that is, $\mathbb{U}(x)=x$. By default, we set $h=1, m=0.15$. Based on these settings we calculate the default $s_{\text {opt }}=1.6$.

We examine three approaches;

- PRA-B - peak rate admission using binary shaping. The system admits APs based on their peak demands and assigns channels to APs statically, and $r_{n} \in\{0,1\}$. We apply the local coordination mechanism [4] to determine $r_{n}$ and spectrum allocations.

- PRA-C - peak rate admission using continuous shaping. It is the same as PRA-B except $r_{n} \in[0,1]$. We apply the topology-based optimization [21] to determine $r_{n}$ and spectrum allocations.

- SPARTA-B - SPARTA with binary shaping.

- SPARTA-C - SPARTA with continuous shaping.

- No Adm - no admission control and APs contend for spectrum in each time slot.

We use two performance metrics. Spectrum utilization refers to the total amount of demand supported per time slot. Node outage rate refers to the probability of outage for a node. In a time slot, an admitted node experiences an outage if its achieved spectrum usage in a time slot is less than its demand.

\section{A. Effective Rate vs Peak Rate Admission Control}

We compare the spectrum utilization of SPARTA and PRA, when APs have uniform spectrum demand statistics. We examine both binary and continuous shaping policies. We note that PRA optimizes based on the original interference constraints, while SPARTA optimizes over the simplified Node-L constraints. Next, we show that despite the Node$\mathrm{L}$ constraints are more restrictive, SPARTA still achieves significant improvements over PRA.

Figure 3 shows the spectrum utilization as a function of the network size. Both SPARTA and PRA result in loss of spectrum utilization compared to the system without admission control. However, they can reliably guarantee the outage rate to below $2 \%$ while the system without admission control suffers from an average outage rate of $60 \%$ for a network size of 500 and $20 \%$ for 300 . Clearly there is a fundamental tradeoff between stability and spectrum utilization. Furthermore, compared to PRA, SPARTA increases spectrum utilization by $80-100 \%$. 


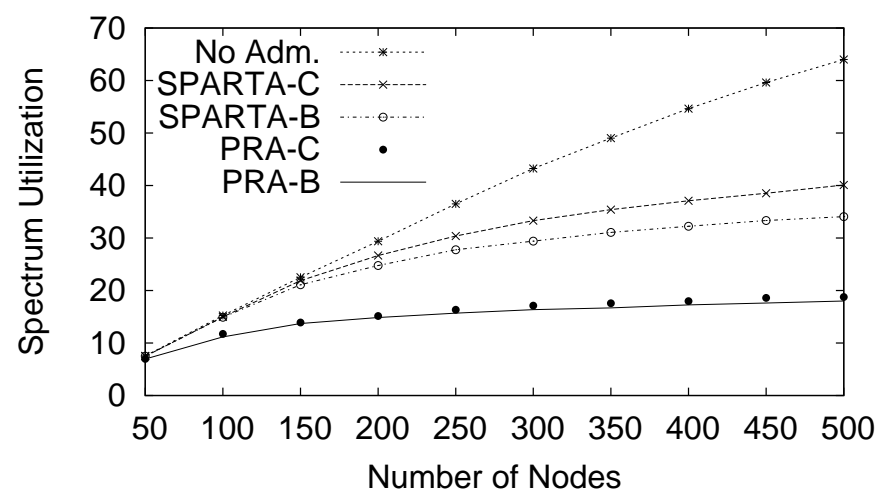

Fig. 3. Spectrum utilization of different shaping schemes.

In the same graph we also compare the performance of the binary and continuous spectrum shaping. Continuous shaping is slightly better because of the increase in granularity. We note that as the network size increases, the difference between the two shaping policies becomes more visible for SPARTA. This is because continuous shaping allows a higher degree of multiplexing, and hence the impact of granularity becomes more visible.

\section{B. Impact of Shaping Aggressiveness}

To verify the efficiency of SPARTA's demand shaping algorithms, we define a notion of admission aggressiveness. A system has an admission aggressiveness of $u$ if it admits demands that are $u$ times the demands derived by the admission algorithm. For a given aggressiveness of $u$, we examine the actual outage rate $p(u)$. A demand shaping algorithm is efficient if $\operatorname{argmax}_{u}\{p(u) \leq p\} \rightarrow 1$ where $p$ is the target outage rate used by the admission algorithm.

Figure 4 examines the CDF of node outage rate for SPARTA under different values of $u$ for $\gamma=2$ and $\gamma=3$, corresponding to target outage rates of $e^{-2}=0.13$ and $e^{-3}=0.05$. We see that SPARTA is highly efficient for $\gamma=3, \operatorname{argmax}_{u}\{p(u) \leq$ $p\} \approx 1.2$; but becomes weaker for $\gamma=2$. This is because the formulation of effective rate tends to over-estimate the outage probability, especially for small $\gamma$ values. Previous studies on wired networks have observed a similar trend [17].

\section{Impact of Non-Uniform Demand Patterns}

We examine the use of uniform $s$ when spectrum demands have non-uniform statistics. In particular, we assume that each AP's peak demand rate $h$ and mean demand rate $m$ are uniformly distributed between $[H 1, H 2]$ and $[R 1, R 2]$, respectively. For SPARTA, we compare two options to compute the $s$, a) an exhaustive search of the optimal $s$ to maximize the spectrum utilization, and b) a heuristic based $s$ using the medians of the peak rate and mean rate in the range of $[H 1, H 2]$ and $[R 1, R 2]$.

For a total capacity of 10 and a deployment of 1000 APs, Figure 5 compares the spectrum utilization under five combinations of On/Off demand patterns listed in Table II. Results
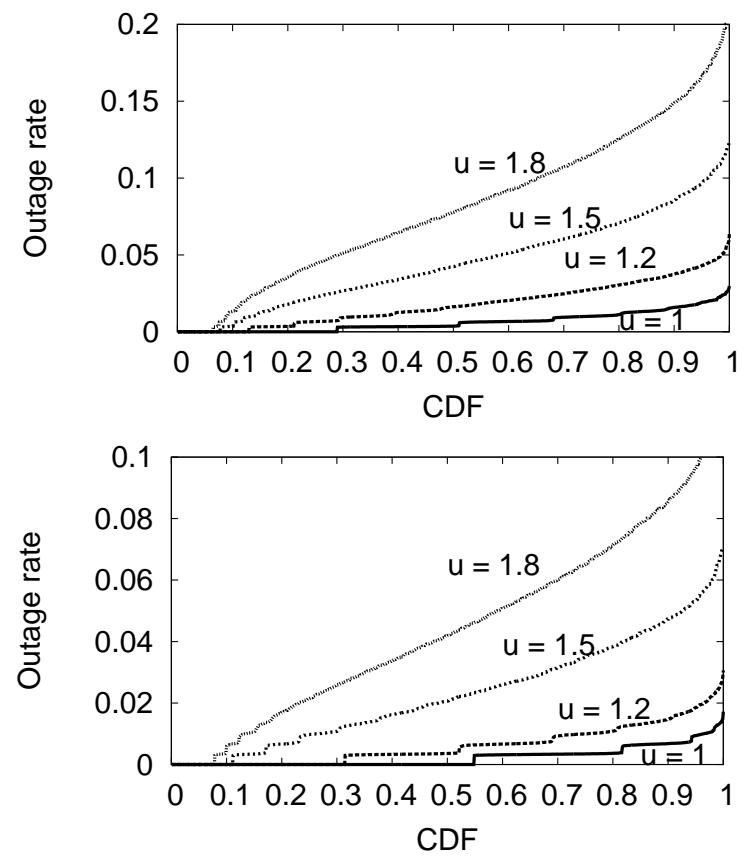

Fig. 4. CDF of node outage rate for different shaping aggressiveness: (upper) $\gamma=2$, (lower) $\gamma=3$.

in Figure 5 shows that both SPARTA approaches achieve significant improvement over PRA, as high as 60\%. More importantly, the performance of the heuristic $s$ is very close to the optimal $s$, making it favorable for its low-complexity. We also observe that for a given peak rate range, lowering the mean rate leads to higher gain in SPARTA, because of the increased degree of multiplexing.

\section{Impact of Non-ON-OFF Demand Patterns}

We examine the performance of SPARTA and PRA when spectrum demands follow the semi-exponential model. The probability density function and the calculated effective rate for semi-exponential demand are

$$
\begin{gathered}
f(x)=\left\{\begin{array}{rll}
\frac{\lambda}{1-e^{\lambda h}} e^{-\lambda x} & : & x \in[0, h] \\
0 & : & x \in(h,+\infty)
\end{array}\right. \\
\alpha(s)=\frac{1}{s} \log \left(\frac{\lambda}{\left(1-e^{-\lambda h}\right)(s-\lambda)}\left(e^{(s-\lambda) h}-1\right)\right)
\end{gathered}
$$

where $h$ is the peak rate of the demand and $\lambda$ decides the burstyness of the demand. Given $h$, a larger $\lambda$ will result in a lower mean rate. We set $h$ and $\lambda$ uniformly distributed in $[\mathrm{H} 1, \mathrm{H} 2]$ and $[\lambda 1, \lambda 2]$, as shown in Table III. We compute the heuristic $s$ using the median of $[\mathrm{H} 1, \mathrm{H} 2]$ and $[\lambda 1, \lambda 2]$. Figure 6 compares the performance of SPARTA and PRA. Similar conclusions are drawn.

\section{E. Impact of Network Topology}

To examine the impact of network topology, we evaluate SPARTA and PRA under both random and clustered topologies. The clustered topology simulates a "hot-spot" area where a large number of APs are placed in a small area such as a city center. We produce the cluster by first deploying a cluster 
TABLE II

TRAFFIC CHARACTERISTICS IN DIFFERENT DATA SETS.

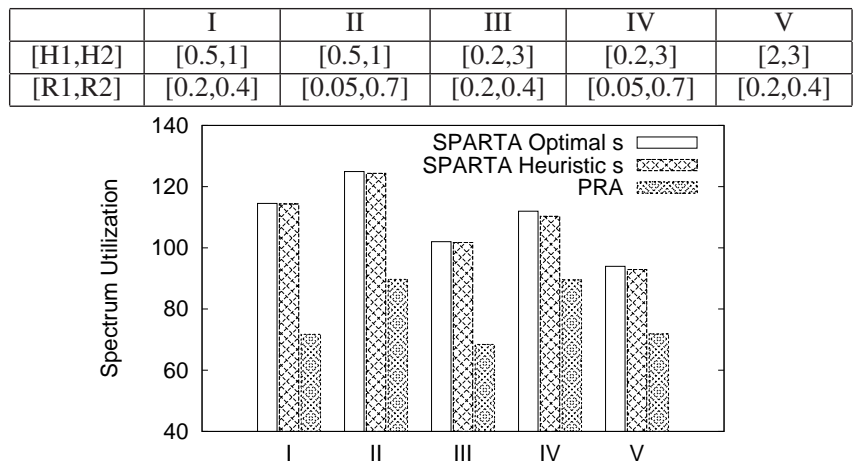

Fig. 5. Comparison of SPARTA and PRA under different traffic characteristics, assuming On/Off demand patterns.

sub-area of radius 0.4 on the $1 \times 1$ area, with $w$ nodes in the cluster area and $1000-w$ nodes uniformly over the entire area, as shown in Figure 7. By varying $w$, we can examine the impact of the clustering degree on the system performance. Figure 7 illustrates the spectrum utilization under different cluster degree $w$. As $w$ increases, the spectrum utilization decreases because the level of interference increases. Similarly, SPARTA improves the spectrum utilization significantly.

\section{Practical Considerations}

In this section, we discuss several practical issues for implementing SPARTA.

Identifying Interference Constraints - The server performing demand shaping needs to collect the global interference constraints. There are multiple mechanisms to obtain this information. We list three complementary mechanisms.

(1) The server performs network measurements to collect interference constraints. Cellular networks use a similar mechanism to examine interference conditions among base stations.

(2) Individual nodes scan radio signals to find interfering nodes and report their findings to the server.

(3) Clients associated with nodes sense radio signals and provide feedback on findings of interfering nodes [18]. This mechanism has been shown to help refine the interference map.

Identifying Demand Statistics- The proposed solution also requires information on demand statistics. We argue that this is possible because nodes who request spectrum are mostly components from infrastructure networks, such as cellular and WiMAX base stations, mesh access points. Most networks perform network planning to optimize node placement and resource allocation. When demand statistics are multi-timescale, nodes can report different effective-rate statistics across time and re-negotiate their spectrum demands. Similar approaches have been verified in the context of wired networks [17].

\section{CONCLUSION}

In this paper, we consider the problem of providing stable and efficient spectrum access in dynamic spectrum networks. We propose SPARTA, a new DSA architecture that treats
TABLE III

TRAFFIC CHARACTERISTICS IN SEMI-EXPONENTIAL PATTERNS.

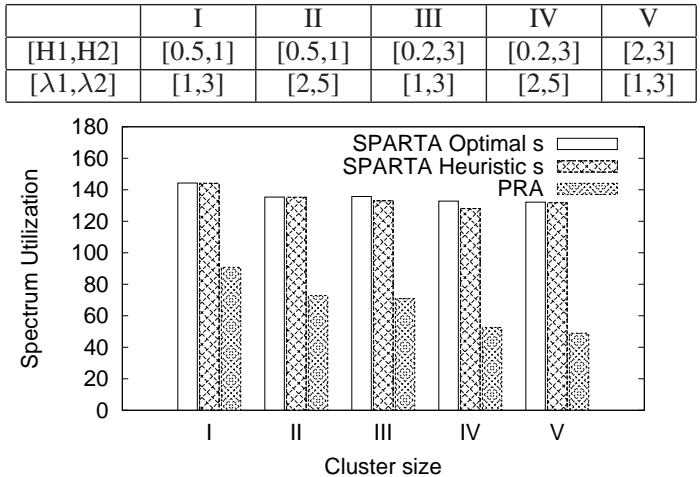

Fig. 6. Comparison of SPARTA and PRA under semi-exponential demand patterns.

stability and efficiency as the first priority. SPARTA combines proactive planning with reactive adaptation to match spectrum allocation to time-varying demands. Given a target outage rate, SPARTA introduces an effective-rate based admission control component to allow time and spatial multiplexing of spectrum demands, and a distributed coordination based spectrum allocation component to adapt instantaneous spectrum allocation to match time-varying demands. Using both analytical verification and experimental simulation, we show that SPARTA can guarantee stable spectrum access while maximizing spectrum utilization. Our future work is to refine and experiment SPARTA designs using measured spectrum demand traces.

\section{REFERENCES}

[1] A. Berger, W. W. Extending the effective bandwidth concept to networks with priority classes. IEEE Communications Magazine 36 8 (1998), 78-83.
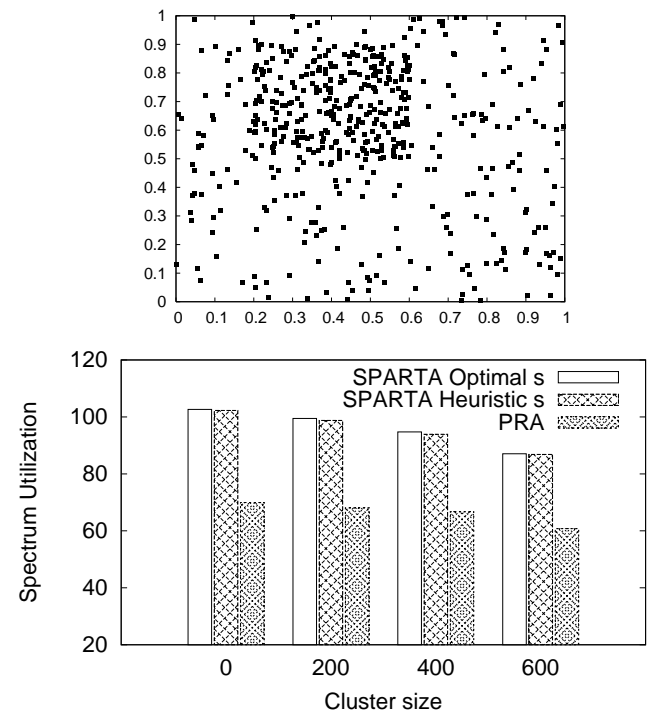

Fig. 7. (upper) Sample topology of a clustered network; (lower) Comparison of SPARTA and PRA in a clustered network. 
[2] Akyildiz, I. F., LeE, W. Y., Vuran, M., And Mohanty, S. NeXt generation/dynamic spectrum access/cognitive radio wireless networks: A survey. Computer Networks Journal (Elsevier) (2006).

[3] Buddhikot, M. M., AND Ryan, K. Spectrum management in coordinated dynamic spectrum access based cellular networks. In Proc. of IEEE DySPAN (November 2005).

[4] CAO, L., AND ZHENG, H. Spectrum allocation in ad hoc networks via local bargaining. In Proc. of SECON (September 2005).

[5] CAO, L., AND Zheng, H. SPARTA: Stable and efficient spectrum access in next generation dynamic spectrum networks: Extended version. Tech. rep., University of California, Santa Barbara, 2008.

[6] Courcoubetis, C., Kelly, F., AND Weber, R. Measurement-based usage charges in communication networks. In Operations Research, vol. 48. 2000, pp. 535-548.

[7] EtKin, R., PAReKh, A., AND Tse, D. Spectrum sharing for unlicensed bands. In Proc. of IEEE DySPAN (November 2005).

[8] Gandhi, S., Buragohain, C., CaO, L., Zheng, H., and Suri, S. A general framework for wireless spectrum auctions. In Proc. of IEEE DySPAN (2007).

[9] GibBens, R. Traffic characterisation and effective bandwidths for broadband network traces. Tech. rep., Statistical Laboratory Research Report, University of Cambridge, 1996-9.

[10] Guerin, R., Ahmadi, H., And Naghshineh, M. Equivalent capacity and its application to bandwidth allocation in high-speed networks. IEEE Journal on Selected Areas in Communications 9 (1991), 968-981.

[11] Huang, J., Berry, R., AND Honig, M. Auction mechanisms for distributed spectrum sharing. In Proc. of 42nd Allerton Conference (September 2004)

[12] Jain, K., Padhye, J., Padmanabhan, V., And Qiu, L. Impact of interference on multi-hop wireless network performance. In Proc. of MobiCom (2003).

[13] Jain, K., Padhye, J., V.N.Padmanabha, and Qiu, L. Impact of interference on multi-hop wireless network performance. In Proc. of MobiCom (September 2003).

[14] Kamakaris, T., Buddhikot, M. M., And IYer, R. A Case for Coordinated Dynamic Spectrum Access in Cellular Networks. In Proc. of IEEE DySPAN (November 2005).

[15] Kelly, F. Notes on effective bandwidths. In Stochastic Networks: Theory and Applications, F. Kelly, S. Zachary, and I. Ziedins, Eds., Royal Statistical Society Lecture Notes Series. Oxford University Press, 1996, pp. 141-168.

[16] Kelly, F. P. Charging and accounting for bursty connections. In Internet economics. MIT Press, Cambridge, MA, 1997, pp. 253-278.

[17] KNightly, E., AND Shroff, N. Admission control for statistical qos: Theory and practice. IEEE Network 13, 2 (1999), 20-29.

[18] Mishra, A., Brik, V., Banerjee, S., Srinivasan, A., ANd ArBAUGH, W. A client-driven approach for channel management in wireless LANs. In Proc. of IEEE Infocom (2006).

[19] NEEL, J., AND REED, J. Performance of distributed dynamic frequency selection schemes for interference reducing networks. In Proc. of Milcom (October 2006).

[20] NiE, N., AND Comaniciu, C. Adaptive channel allocation spectrum etiquette for cognitive radio networks. In Proc. of IEEE DySPAN (November 2005).

[21] Peng, C., Zheng, H., And Zhao, B. Y. Utilization and fairness in spectrum assignemnt for opportunistic spectrum access. Mobile Networks and Applications (MONET) 11 (May 2006), 555-576.

[22] Xing, Y., Chandramouli, R., Mangold, S., and Shankar, S. Dynamic spectrum access in open spectrum wireless networks. IEEE Journal on Selected Areas in Comm. 24, 3 (March 2006), 626-637.

[23] Yuan, Y., Bahl, P., Chandra, R., Chou, P. A., Ferrell, J. I., Moscibroda, T., Narlanka, S., and Wu, Y. Knows: Kognitiv networking over white spaces. In Proc. of IEEE DySPAN (2007).

[24] Yuan, Y., Bahl, P., Chandra, R., Moscibroda, T., Narlanka, S., AND WU, Y. Allocating dynamic time-spectrum blocks in cognitive radio networks. In Proc. of MobiHoc (2007).

[25] Zheng, H., AND CaO, L. Device-centric spectrum management. In Proc. of IEEE DySPAN (November 2005).

\section{APPENDIX}

\section{A. Proof of Theorem 1}

We prove Theorem 1 by showing the fact that by properly taking a uniform $s$ the constraint clause in (7) is maximally loosen. In other words, if there exists a set of $r_{n}$ which can satisfy the constraint in (7) by taking a set of $s_{n}$, then this set of $r_{n}$ can also satisfy the constraint in (7) by taking all $s_{n}=\dot{s}$. Therefore by taking a uniform $s$ we maintain the optimality of the solution to (7).

When node traffics are uniform, i.e., $\alpha_{i}()=.\alpha($.$) . We can$ rewrite the constraint in the optimization problem (7) as

$$
\exists s_{n}, \quad \sum_{i \in I_{n}} \alpha^{r_{i}}\left(s_{n}\right) \leq C-\frac{\gamma}{s_{n}}, \quad \forall 1 \leq n \leq N
$$

Because $r_{i} \in\{0,1\}$, the constraint reduces to

$$
\sum_{i \in I_{n}} r_{i} \leq \frac{C-\frac{\gamma}{s_{n}}}{\alpha\left(s_{n}\right)}, \quad \forall 1 \leq n \leq N
$$

Here $\sum_{i \in I_{n}} r_{i}$ is the number of admitted nodes that are in constraint $I_{n}$. We can see that since the right side of the above equation only depends on $s_{n}$, we can optimally loosen the above constraint by taking a $s_{n}$ which maximizes the right side. Note that this optimal value of $s_{n}$ does not depend on $n$. Therefore, to optimize (7), a uniform optimal $s$ is sufficient for uniform node traffics.

We can see that $s$ only depends on $C, \alpha($.$) , and \gamma$, and does not depend on the topology of the network.

\section{B. Proof of Theorem 2}

Proof: For simplicity we prove the theorem in the case that there are only two types of different traffics, denoted $t_{1}$ and $t_{2}$. It's straightforward to extend it for $J>2$.

For any constraint in the form as (7), we prove that if there is a $s$ which is not in the range $\left[\min \left\{\dot{s}_{1}, \dot{s}_{2}\right\}, \max \left\{\dot{s}_{1}, s_{2}\right\}\right]$, and the constraint holds, then we can replace $s$ with a $s^{\prime}$ in the range $\left[\min \left\{\dot{s}_{1}, \dot{s}_{2}\right\}, \max \left\{\dot{s}_{1}, \dot{s}_{2}\right\}\right]$ such that the constraint still holds. This means that under no cases do we need to pick up a $s$ value from outside the range $\left[\min \left\{\dot{s}_{1}, s_{2}\right\}, \max \left\{\dot{s}_{1}, \dot{s}_{2}\right\}\right]$. In other words, the optimal $s$ value can be found in the range $\left[\min \left\{\dot{s}_{1}, \dot{s}_{2}\right\}, \max \left\{\dot{s}_{1}, \dot{s}_{2}\right\}\right]$.

The constraint can be written as

$$
k_{1} \alpha_{1}(s)+k_{2} \alpha_{2}(s) \leq C-\frac{\gamma}{s}
$$

where $k_{1}$ and $k_{2}$ are the number of nodes having traffic $t_{1}$ and $t_{2}$, respectively. For any $s<\min \left\{s_{1}, \dot{s}_{2}\right\}$ (the case $s>$ $\max \left\{s_{1}, s_{2}\right\}$ can be treated similarly), we can show that $s_{1}$ can replace $s$ and the constraint still holds, i.e.

$$
k_{1} \alpha_{1}\left(\dot{s}_{1}\right)+k_{2} \alpha_{2}\left(\dot{s}_{1}\right) \leq C-\frac{\gamma}{\dot{s}_{1}} .
$$

From the property of effective rate we can prove that

$$
\frac{C-\gamma / \dot{s}_{1}}{\alpha_{1}\left(\dot{s}_{1}\right)} \geq \frac{C-\gamma / s}{\alpha_{1}(s)} \text { and } \frac{C-\gamma / \dot{s}_{1}}{\alpha_{2}\left(\dot{s}_{1}\right)} \geq \frac{C-\gamma / s}{\alpha_{2}(s)}(20)
$$

Put (20) into (18) we can get (19). 


\section{Proof of Theorem 3}

Proof: We can prove this result by proving the following property: "If a constraint $n$ is satisfied by choosing $s_{n}$, i.e.,

$$
\sum_{i \in I_{n}} \alpha_{i}\left(s_{n}\right) \leq C-\frac{\gamma}{s_{n}}
$$

then it can also be satisfied by choosing $s$ and increasing the capacity to $C / g(s)$."

By showing this property, it is straightforward to show that because any satisfiable constraint can be satisfied by a uniform $s$ and capacity $C / g(s)$, any achievable utilization value can be achieved by a uniform $s$ and capacity $C / g(s)$.

From (21),

$$
\begin{aligned}
1 & \geq \sum_{i \in I_{n}} \frac{\alpha_{i}\left(s_{n}\right)}{C-\gamma / s_{n}} \geq \sum_{i \in I_{n}} \frac{\alpha_{i}\left(\dot{s}_{i}\right)}{C-\gamma / \dot{s}_{i}} \\
& =\sum_{i \in I_{n}} \frac{\alpha_{i}(s)}{C-\gamma / s} \cdot g_{i}(s) \geq g(s) \cdot \sum_{i \in I_{n}} \frac{\alpha_{i}(s)}{C-\gamma / s} . \\
& \geq \sum_{i \in I_{n}} \frac{\alpha_{i}(s)}{C / g(s)-\gamma / s},
\end{aligned}
$$

which is equal to

$$
\sum_{i \in I_{n}} \alpha_{i}(s) \leq C / g(s)-\gamma / s .
$$

